\title{
Im Spannungsfeld zwischen "case history» und Spiritualität
}

\section{Michel Lansel-Schellenberg}

Dr. med. et lic. theol., Facharzt für Psychiatrie und Psychotherapie

Es gibt Erscheinungen, die gewissermassen zeitlos sind und die bis heute eine grosse Anziehung ausüben können. Hierzu gehören etwa die antiken Mysterien sowie die antike Tempelmedizin. Auch wenn keine direkten Linien zu mystischen und spirituellen Erfahrungen unserer Zeit nachgezeichnet werden können, so ist gewiss von einer inneren Verwandtschaft auszugehen. Mysterien waren schon immer von einer geheimnisvollen, «mysteriösen» Aura umgeben. Sie wurden in der Antike mit einem strengen Schweigegebot belegt, galten aber auch als unaussprechliche innere Erfahrung, die «auf das ganze weitere Leben" ausstrahlen und die menschliche "Existenz» verwandeln sollte [1]. «Die Schau, epopteia, [...] als höchste Form der Einweihung bezeichnet, bringt einen zentralen Vorgang [...] auf den Begriff: das Anschauen, sich Versenken, Stille Werden angesichts heiliger Gegenstände und Rituale als kontemplative und letzte Stufe, sich dem Göttlichen zu nähern» [2]. Zahlreiche Mythen prägten die verschiedenen Mysterienspiele. Als zentrales Element galt der Verlust oder Tod und das Wiederauffinden bzw. die Erweckung der verehrten Gottheit. So folgte etwa der Trauer über den Raub der Persephone durch Hades in den Eleusinischen Mysterien ihre bejubelte Wiederkunft, und der Isiskult findet seinen feierlichen Abschluss mit der Wiederbelebung und dem Auffinden des durch Seth zerstückelten Osiris [1].

Menschliche Kontingenz, mit anderen Worten die Zufälligkeit und Vergänglichkeit unserer Existenz, soll durch einen Akt des Heils, der Trost und Sicherheit vermitteln will und an dem alle Anwesenden Anteil haben, überwunden werden. Über das Gemeinschaftserlebnis hinaus scheint die Vertiefung der Erlebensdimension im Vergleich zu anderen Kulten jener Zeit das eigentlich Neue zu sein [1].

In der Initiationsfeier, wie sie uns der Dichter Plutarch überliefert, trat der Myste aus dem Dunkel ans Licht. Dieser «erhellende» Moment lässt sich psychologisch auch als Metapher für einen Akt der Bewusstwerdung deuten. Dies «weist offenbar auf jene Stufe in der Entwicklung der Gesellschaft und des Bewusstseins hin, die man mit Begriffen wie «Entdeckung des Geistes` und «Entdeckung der Persönlichkeit` charakterisiert hat» [1]. Dieses neuartige Bedürfnis, die Welt aus der Innenperspektive heraus wahrzunehmen und $\mathrm{zu}$ deuten, wurde von der sogenannten Tempelmedizin, einer Bewegung, die sich ab dem fünften Jahrhundert v.Chr. im hellenischen Raum ausbreitete [4], aufgenommen und vertieft.

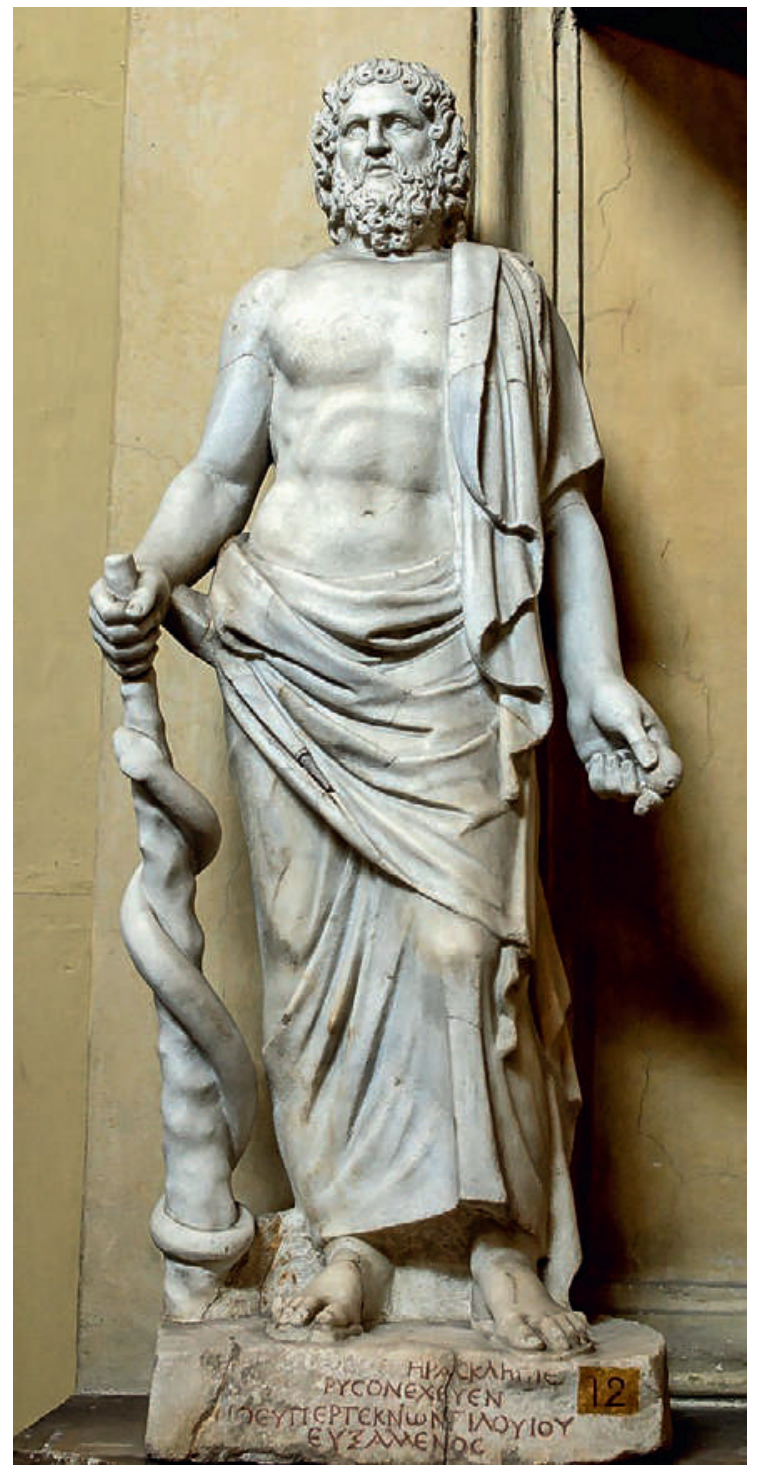

Asklepios, römische Marmorstatue, Kopie eines griechischen Originals aus dem 5. Jahrhundert. 
Dieses neue Phänomen weist zahlreiche Berührungspunkte mit den Mysterien auf, etwa die «Erwartung eines bestimmten, grossen Erlebnisses am Ziele» [5], wobei das griechische Wort telos nicht nur Ziel, sondern auch Fest oder Weihe bedeuten kann [1]. Des Weiteren sind kultische Analogien, insbesondere der Aspekt der Geheimhaltung, zu nennen. Auch wenn man deswegen heute nur wenige Einzelheiten kennt, zeigen die "mythologischen Ausdrucksweisen" beider Erscheinungen doch «bedeutsame Übereinstimmungen» [5]. So spielt etwa die Schlange - das charakteristische Attribut des Gottes Asklepios [5, 6] - auch in den Mysterien eine bedeutsame Rolle, wo sie als ein «chtonisches» (erdbezogenes) Symbol eine Affinität zur Unterwelt aufweist [vgl. 5, 6]. In der Tempelmedizin kann sie in der Vorstellung der Kultteilnehmer während des Tempelschlafes [6], der Inkubation, als heilende Traumgestalt (s.u.) erscheinen. Wie aber muss man sich diese Asklepiosheiligtümer vorstellen? Blenden wir hierzu ins achte vorchristliche Jahrhundert, in homerische Zeiten, zurück. Damals kannten die Griechen zwei Heiltraditionen. Zum einen jene des Feldarztes, des «heilkundigen Heerführers", wie sie etwa in der Ilias belegt ist und die den Arzt der Frühzeit kennzeichnet [4]. Zum anderen vollzog sich «die Ausweitung der Medizin zum eigentlichen Gewerbe, das Aufkommen des berufstätigen Arztes, der [...] gegen Entgelt der Allgemeinheit zur Verfügung steht [...] im Rahmen der aufstrebenden [...] Polis» [4].

Darüber hinaus gab es Bemühungen, die ärztliche Tätigkeit rational zu fundieren. So lassen sich bereits im späten sechsten Jahrhundert v.Chr. kritische Untersuchungen über die Ursache und Behandlung von Er-

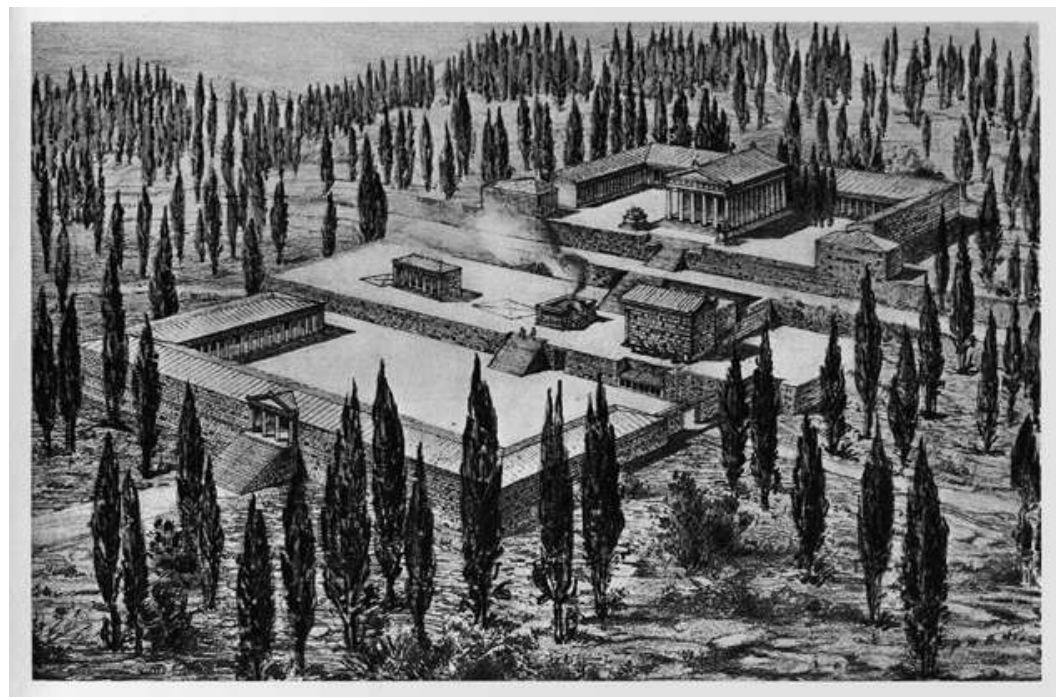

Abbildung 1: Rekonstruktion des Asklepieion von Kos aus hellenistischer Zeit (Aus: Kerényi K. Der göttliche Arzt. Zürich: Konzett \& Huber; 1948). krankungen nachweisen - man würde heute durchaus von "case histories» sprechen [7]. In diesem Prozess medizinischer Verwissenschaftlichung nahm der ca. 460 v.Chr. auf der Insel Kos geborene und dem Ärztegeschlecht der Asklepiaden entstammende Hippokrates eine herausragende Stellung ein; zudem sollte er durch sein Wirken das hohe Ansehen der koischen Ärzteschule begründen [6].

Im späten 6. Jh. v.Chr. lassen sich Untersuchungen über Ursache und Behandlung von Erkrankungen nachweisen - «case histories».

Die Asklepiaden bildeten einen «Kultverein [...], dessen Mitglieder Asklepios», den Sohn Apollos, «als ihren mythischen Ahnherren betrachteten" [6]. Diese mythologische Genealogie hatte vermutlich den ganz konkreten Sinn, die standesethischen Prinzipien quasi metaphysisch zu begründen. Dadurch sollte das Vertrauen der Kranken in die lautere Gesinnung des Arztes bestärkt werden. Mit dem auf «Apollon dem Arzt und Asklepios und Hygieia und Panakeia» geleisteten Schwur verpflichtete sich der Arzt vertraglich, die im Eid festgelegten Behandlungsnormen immer zu erfüllen und so «zum Nutzen der Kranken» zu handeln [8].

Nach dem Tode von Hippokrates 377 v.Chr. wurde in einem heiligen Hain auf Kos ein Asklepiosheiligtum gegründet, was wohl unter dem Einfluss der Kultstätte in Epidauros geschah (Abb. 1). Es gibt gewisse, allerdings nur schwach belegte Hinweise, dass damit eine Wendung zu neuer religiöser Tiefe eingeleitet wurde [4, 5]. Umgekehrt ist anzunehmen, dass die «stärkere wissenschaftlich-medizinische» Ausrichtung, die «sich später in den meisten Asklepieia bemerkbar machte, überhaupt von Kos ausging» [5]. In welcher Gestalt aber breitete sich diese neue "spirituelle» Bewegung im fünften vorchristlichen Jahrhundert in der hellenischen Welt aus? Ihre Nähe zu den Mysterien wurde bereits erörtert, namentlich die Sprache und Bilder der Mythologie sowie der Geheimhaltungscharakter. Allerdings gibt es einen grundlegenden Unterschied zu beachten: Mysterien wurden kollektiv gefeiert und stellten das grosse zyklische Geschehen von Tod und Leben, von Werden und Vergehen, szenisch dar. Im Asklepieion dagegen suchte der Patient die Gottheit «auf eine viel persönlichere Weise» [5] auf. Dabei dürfte sich die Zeremonie etwa so abgespielt haben: Wohl im Anschluss an rituelle Waschungen oder Salbungen zog sich der Patient allein in das innere Gemach bzw. die Schlafhalle (gr. enkoimeterion) zum Tempelschlaf zurück [4-6]. In der Stille und Abgeschiedenheit wurden vermutlich 


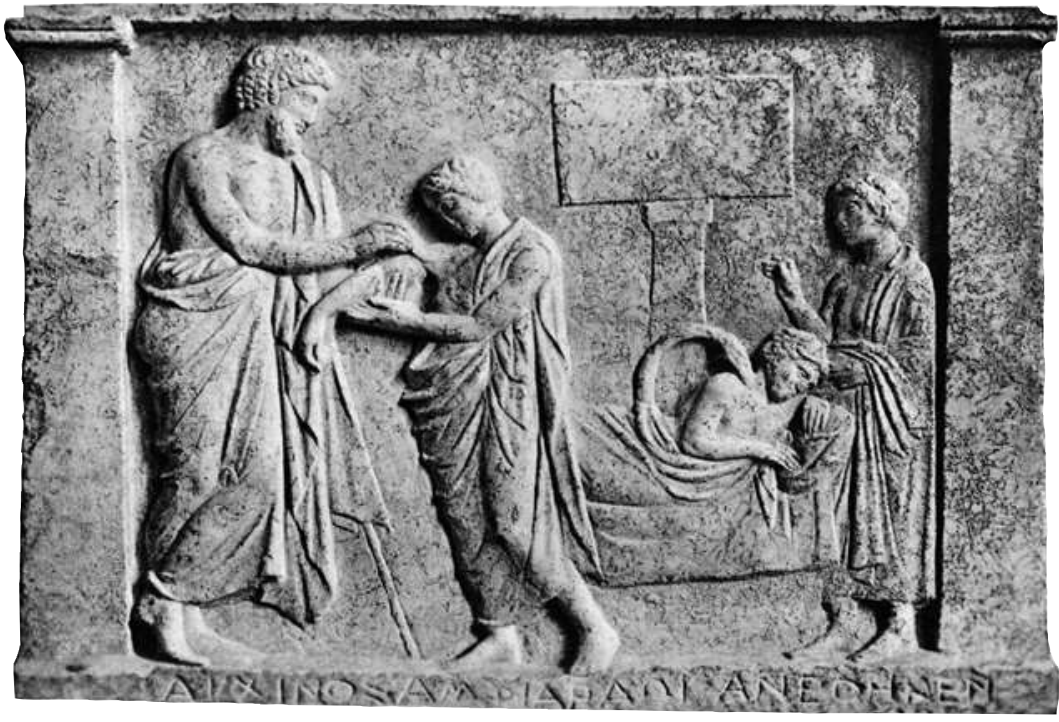

Abbildung 2: Weihrelief des Archinos an Amphiaraos, den in Oropos (Attika) verehrten Orakel- und Heilgott. Athen, Nationalmuseum.

sowohl Selbstwahrnehmung wie auch Selbstreflexion geschärft. Die sakrale Aura des Tempels dürfte überdies mystisches und spirituelles Erleben begünstigt haben. Schliesslich gab man sich im Schlaf ganz der Sprache und den Bildern des «Unterbewussten» hin. Diese wurden, da der bewussten Einflussnahme entzogen, wohl als unabweislich erlebt. Dabei spielte in der klassischen Periode die «direkte Gottesbegegnung im Traumgesicht» eine entscheidende Rolle [6]. «In der römischen Kaiserzeit» dagegen «gewinnt die Mitteilung eines Orakelspruchs an Bedeutung, der nach dem Erwachen des Adoranten ausgelegt werden muss" [6]. Aus dieser Sicht könnte man zwar von einer Vorstufe von Psychotherapie sprechen - von einer tiefenpsychologischen Behandlung im Sinne einer langdauernden Analyse mit dem Ziel eines Bewusstwerdungsprozesses im heutigen Sinne kann jedoch noch keine Rede sein. Zahlreich sind die in Votivgaben bezeugten Wunderheilungen. Auch "unfruchtbare Frauen» suchten das Asklepieion auf, «um vom Gotte schwanger zu werden: 〈Nikesibule von Messene〉 - so lautet [...] ein 〈Heilungsbericht -, schlief um Kindersegen zu erhalten, im Heiligtum und sah einen Traum. Es träumte ihr, der Gott sei mit einer Schlange, die ihm folgte, zu ihr gekommen, mit dieser habe sie verkehrt. Und darauf empfing sie übers Jahr zwei Knaben»» [zit. in 5].

Bekannt ist die Darstellung auf einem Relief, das dem Orakelgott Amphiaraos nach erfolgter Heilung geweiht wurde (Abb. 2). Seine Ikonographie unterscheidet sich nicht von jener der Asklepiosheiligtümer.
Die Heilung ist offenbar doppelt dargestellt. Folgende Deutung erscheint plausibel: Im Vordergrund links steht der Arztgott, der dem Kranken die Schulter massiert oder eine Salbe mit einem Spachtel aufträgt. Die Säule im Hintergrund rechts könnte das stilisierte innere Gemach, in dem sich der Kranke zum Tempelschlaf zurückgezogen hatte, darstellen. Im Traum würde ihm die Gottheit in Gestalt einer Schlange, die die Schulter mit ihrer heilenden Zunge leckt, erscheinen. Ganz rechts stünde schliesslich der Genesene, der zum Dank das Weihrelief stiftete*.

Doch nicht alle Patienten dürften eine vergleichbare Heilung erfahren haben. Rund zehn Jahre lang soll sich etwa der Redner P. Aelius Aristeides (117-187 n.Chr.) im Asklepieion von Pergamon aufgehalten haben, wo er im «Zwiegespräch mit dem Gott sein krankheitsgeplagtes Leben zu meistern suchte» [4|. Schliesslich gilt es noch, die Orte, an denen Asklepieia errichtet wurden, zu beachten. In Kos findet sich die Tempelanlage etwa eine Gehstunde von der Hauptstadt «landeinwärts, wo die Insel gebirgig zu werden beginnt» [5]. Auf einer "milden und gesunden Anhöhe» mit wunderbarem Blick aufs Meer und «nicht weit entfernt von einer mineralischen Quelle» [5] durfte die geplagte Seele wieder zu sich finden und der

\section{Das Bedürfnis, die Welt aus der Innen-}

\section{perspektive heraus zu deuten, wurde von der} Tempelmedizin vertieft.

Kranke Heilung oder zumindest Linderung erfahren. Und wer das Asklepieion heute besucht, sollte sich nicht nur von der Aura des Ortes betören lassen, sondern sich auch die Zeit nehmen, die Umgebung etwas zu erkunden; denn hinter der Tempelanlage, wo ein lichter Pinienwald steht, wächst eine seltene Orchidee, die aufzusuchen sich lohnt. Womöglich war sie schon Hippokrates aufgefallen; sie heisst sinnigerweise Orchis sancta.

\section{Literatur}

1 Burkert W. Antike Mysterien. 4. Aufl. München: C.H. Beck; 2003.

2 Kloft H. Mysterienkulte der Antike. 3. Aufl. München: C.H. Beck; 2006

3 Friedlein C. Geschichte der Philosophie. 13. Aufl Berlin: Erich Schmidt. 13;1980.

4 Koelbing HM. Arzt und Patient in der antiken Welt. Zürich/München: Artemis Verlag; 1977.

5 Kerényi K. Der göttliche Arzt. Studien über Asklepios und seine Kultstätten. Zürich: Conzett \& Huber; 1948.

6 Leven K.-H. (Hrsg.). Antike Medizin. Ein Lexikon. München: C.H. Beck; 2005 .

7 Lloyd G. E. R. Hippocratic Writings. London: Penguin Books; 1983

8 Hippokrates. Der Eid des Arztes. Frankfurt am Main/Leipzig: Insel; 1996 